\title{
The Beneficial Effects of Regular Physical Activity Intradialysis
}

\author{
Lillana P Alemán* and Héctor Pérez G \\ Department of Nephrology, National Institute of Cardiology, Mexico
}

*Corresponding author: Lillana P Alemán, Nephrology Department, National Institute of Cardiology, Mexico City, USA.

To Cite This Article: Lillana P Alemán, The Beneficial Effects of Regular Physical Activity Intradialysis. 2020 - 8(2). AJBSR.MS.ID.001244.

DOI: $10.34297 /$ AJBSR.2020.08.001244.

Received: 阱 March 02, 2020; Published: 畊 March 13, 2020

Keywords: Physical activity, Intradialysis, Sarcopenia, Nutrition, Quality of life, Functionality, Hemodialysis, Metabolical, Muscular mass, Chronic

Kidney Disease, Intradialytic hypotension, Physiological

\section{Opinion}

Since 1980's Goldberg demonstrate de benefits of exercise for hemodialysis patients as an adjuvant therapy improving metabolical state [1] and beginning a trend among hemodialysis units initially as an outpatient activity and later during dialyisis. Along time it has been demonstrated in randomized clinical trials the multiple beneficial effects of physical activity, attenuating the catabolic effects associated to hemodialysis and Chronic Kidney Disease (CKD) and improving general wellbeing among this population; it is a well-known fact that patients undergoing dialysis experiment a significant reduction in muscular mass, similar to elderly patients in whom muscular tissue is replaced by fat tissue; this muscular mass wasting correlates with lower functionality as shown by McIntyre in 2006 [2] according to his paper muscle mass, as assessed by the measurement of muscle Cross Sectional Area (CSA) corrected for linear height, was significantly lower in patients receiving dialysis (by about 9\%), as compared with patients with CKD 4 reductions in muscle CSA appeared to be functionally significant.

Muscle CSA was positively correlated with Sit-To-Stands (STS) 60 and negatively correlated with STS 5 assessments of overall physical condition in both males and females (all $\mathrm{P}<0.001$ ). This effect could be palliated by physical activity. In our center in 199517 young patients ( 10 men and 7 women) who were receiving hemodialysis three times per week, were submitted to physical conditioning by pedaling without resistance at 50RPM a Monarck ${ }^{\circledR}$ ergometer for 15 minutes every hour during the 4 hours session performed 3 times a week for 12 weeks. We found that such intensity of exercise did not cause significant changes in blood pressure or heart rate and was well tolerated by patients. On average they recovered 4 kilograms of lean body weight and maintained a controlled pre-HD blood pressure without antihypertensive medications. Interestingly, the recovery of muscle mass was observed in both lower and upper limbs. A control group that did not perform physical activity during the same time period only gained one kilogram of weight [3].

Physical activity also has beneficial effects on functional capacity, anemia, cardiovascular risks factors and on psychosocial problems. In 1986, Painter and colleagues [4] reported the effects of an exercise program that was delivered using a stationary cycle during the dialysis treatment and reported significant improvements in exercise capacity (VO2 peak) and improvements in blood pressure; these results were replicated in other single-center studies. Over time it also has been demonstrated that physical activity can impact increasing phosphorus and urea clearance the pedaling at 60RPM during the whole HDF session in our center improved the extraction of phosphate in $11 \pm 4 \%$, this result has been reproduced several times with different subjects in other centers [5]. Other beneficial effect of physical activity found was the diminishing of drowsiness related to the alkaline load at the beginning of the dialysis, when patients do exercise, they do not fall asleep during treatment regularizing circadian cycle and reducing the need for sleeping drug [6]. It is important to mention that resistance to 
exercise is better during dialysis when compared before or after treatment associated with higher levels of bicarbonate and lower levels of lactic acid. [7]

The hemodynamical effects of intradialytic exercise have been reported extensively: seven trials compared blood pressure differences between patients who did and did not undertake intradialytic exercise. A combined analysis of 287 patients revealed that intradialytic exercise could significantly reduce Systolic Blood Pressure (SBP) (MD -4.87mmHg, 95\% CI -9.20 to $-0.55, \mathrm{p}=0.03$ ) as well as diastolic blood pressure (DBP) (MD $-4.11 \mathrm{~mm} \mathrm{Hg}$, 95\% CI -6.50 to $-1.72, p=0.0007$ ) [8]. On the other hand could also prevent intradialytic hypotension; intradialytic hypotension is reported to occur in $20-30 \%$ of HD treatments and there are not several options to counteract it , first we should review the physiological response to exercise in a healthy cardiovascular system which is characterized by an increased cardiac output achieved through an elevated heart rate and enhanced stroke volume. Sympathetic activation increases heart rate and myocardial contractility leading to higher stroke volume, cardiac output, and arterial pressure.

During submaximal exercise, cardiac output can increase fourfold to match the oxygen demand of skeletal muscle, in patients with ESRD, studies have identified an altered cardiovascular response to submaximal exercise performed off HD. Heart rate and oxygen uptake (VO2) appear to be blunted ( 10\& 45\%, respectively) in comparison to healthy individuals. With exercise during HD, a significant increase in HR and BP was observed ( $\sim 15 \& \sim 13 \%$, respectively) with 30 minutes of low to moderate intensity cycling when compared to standard HD without exercise [8]. Related to these effects on intradialytic hypotension, the impact of exercise in tolerability of ultrafiltration has been measured in our center using the BVM (Blood Volume Monitor) a feature included in 5008 Fresenius Kabi dialysis devices. Patients who performed physical activity experiment a less severe drop in BVM curve compared to those who did not. (Data not published yet). Quality of life is a widely studied aspect in the hemodialysis population and physical activity could improve it with this regard.

In an RCT involving 195 patients reported on the assessment of depression levels at the baseline and endpoint. Within, 111 patients participated in intradialytic exercise, while 84 served as controls. Heterogeneity was found to be significant $(12=77 \%, \mathrm{p}=0.005)$. The random-effects model was used to combine the data. The results showed that intradialytic exercise was able to lower the depression level (SMD -1.16, 95\% CI -1.86 to -0.45) [9]. With this background, during the past 25 years 774 patients aged $32 \pm 12$ years (range 12 to 87 , median 29 years of age),with an average dialysis vintage of $10 \pm 21$ months and $49 \%$ women in our center have performed lowresistance intra-dialytic physical activity throughout the session and in all sessions, . It is important to mention that our population is mostly young and a predominantly nondiabetic $(10 \%$ of the total population).
In addition to patients with diabetes mellitus and patients older than 65 years, the intradialytic exercise program has been introduced to some patients with high cardiovascular risk, including patients with very limited left ventricular ejection fraction and patients with ischemic and valvular disease. In these cases, exercise on hemodialysis is carried out with close cardiovascular monitoring including electrocardiogram, oxygen saturation and central and peripheral skin temperature delta to see the sympathetic nerve activation in response to ultrafiltration. Likewise, patients with physical limitations to exercise such as patients with lower limb amputations or patients with hip fractures due to mineral bone disease, paraplegia or femoral vascular access have been offered alternative exercise programs such as weightlifting.

\section{Conclusion}

In conclusion performing physical activity during hemodialysis can lead to an overall improvement in the well-being of this population, with evidence regarding beneficial effects on cardiovascular disease, the lead cause of death among hemodialysis patients, amelioration of muscular wasting that impacts so profoundly on functionality and even diminishing the effects of depression every hemodialysis center should consider include a physical activity program.

\section{References}

1. Goldberg AP, Hagberg JM, Delmez JA, Haynes ME, Harter HR (1980) Metabolic effects of exercise training in hemodialysis patients. Kidney Int 18(6): 754-761

2. McIntyre CW, Selby NM, Sigrist, M, Pearce LE, Mercer TH, et al. (2006) Patients receiving maintenance dialysis have more severe functionally significant skeletal muscle wasting than patients with dialysisindependent chronic kidney disease. Nephrol Dial Transplant 21(8): 2210-2216.

3. Pérez GH, Socorro R, Jaime HA (1995) Muscle mass increase by exercise training during hemodialysis XIII. International Congress of Nephrology p. 2-6.

4. Painter P (2009) Implementing Exercise: What Do We Know? Where Do We Go? Advances in Chronic Kidney Disease 16(6): 536-544.

5. Manfredini F, Mallamaci F, D’Arrigo G, Baggetta R, Bolignano D, et al. (2016) Exercise in Patients on Dialysis: A Multicenter, Randomized Clinical Trial. J Am Soc Neph 28(4): 1259-1268.

6. Bravo FM, Ramírez AM, Magaña F, De La CG, Flores N, et al. (2007) Intradialytic subjetives sleepness, exercise and metabolic alkalosis. World Congress Nephrol.

7. Verónica G, Rocha I, Ilarraza H, Magaña S, Mariscal A, et al. (2008) Bicarbonate doping in Hemodiafiltration: Effects on submaximal Exercise Test. Blood Purification 26: 115.

8. Vinet A, S Nottin, AM Lecoq, P Obert (2002) Cardiovascular responses to progressive cycle exercise in healthy children and adults. Int J Sports Med 23(4): 242-246.

9. Pu J, Jiang Z, Wu W, Li L, Zhang L, et al. (2019) Efficacy and safety of intradialytic exercise in haemodialysis patients: a systematic review and meta-analysis. BMJ Open 9(1): 020633. 